\title{
Systematic meta-analysis of subsequent pregnancy outcomes in recurrent pregnancy loss couples with parental abnormal chromosomal karyotype
}

\author{
Peng-Sheng Zheng ${ }^{1}$, Shan $\mathrm{Li}^{2}$, and Jing Jing $\mathrm{He}^{2}$ \\ ${ }^{1}$ Affiliation not available \\ ${ }^{2}$ Xi'an Jiaotong University Medical College First Affiliated Hospital
}

October 14, 2021

\begin{abstract}
Background Parental abnormal chromosomal karyotypes are considered as reasons for recurrent pregnancy loss. Objective This systematic meta-analysis evaluated the current evidence on pregnancy outcomes amongst couples with abnormal versus normal chromosomal karyotypes. Search strategy Two independent reviewers screened titles and abstracts identified in EMBASE and PubMed from inception to January 2021. Selection criteria Studies were included if they provided a description of pregnancy outcomes of parental chromosomal abnormality. Data collection and analysis Random effects meta-analysis was used to compare odds of pregnancy outcomes associated with noncarriers and carriers. Main results A significantly lower first pregnancy live birth rate (FPLBR) was found in carriers than in noncarriers with RPL (OR: 0.55; 95\% CI: 0.46-0.65; p<0.00001). Regarding FPLBR between translocation or inversion carriers and noncarriers, a markedly decreased FPLBR was found in translocation (OR: $0.44 ; 95 \%$ CI: $0.31-0.61 ; \mathrm{p}<0.00001)$ but not inversion carriers. The accumulated live birth rate (ALBR) (OR: $0.96 ; 95 \%$ CI: $0.90-1.03 ; \mathrm{p}=0.26$ ) was similar, while the miscarriage rate $(\mathrm{MR})$ of accumulated pregnancies (OR: 2.21; 95\% CI: $1.69-2.89$; $\mathrm{p}<0.00001$ ) was significantly higher in the carriers than in noncarriers with RPL. The ALBR was not significant (OR: $1.82 ; 95 \%$ CI: $0.38-8.71 ; \mathrm{p}=0.45$ ) but the MR (OR: $5.75 ; 95 \%$ CI: $2.57-12.86 ; \mathrm{p}<0.0001$ ) was markedly lower for carriers who choose PGD than natural conception. Conclusions Carriers with RPL had higher risk of miscarriage but obtained a satisfying pregnancy outcome through multiple attempts. No sufficient evidence was found PGD could enhance the ALBR but it was an alternative to decrease the MR.
\end{abstract}

Systematic meta-analysis of subsequent pregnancy outcomes in recurrent pregnancy loss couples with parental abnormal chromosomal karyotype

Shan Li ${ }^{1}$, Jingjing $\mathrm{He}^{1}$, Peng-sheng Zheng ${ }^{1,2}$

1.Department of Reproductive Medicine, The First Affiliated Hospital of Xi'an Jiaotong University, Shaanxi, Xi'an, China.

2. Key Laboratory of Environment and Genes Related to Diseases, Ministry of Education of the People's Republic of China, Shaanxi, Xi'an, China.

*Correspondence:

Peng-Sheng Zheng, M.D., Ph.D.

Director and Professor, Department of Reproductive Medicine,

The First Affiliated Hospital of the Medical College,

Xi'an Jiaotong University, 76 West Yanta Road, Xi'an, Shaanxi Province, 710061, 
The People's Republic of China

Tel: +86-029-82657874 (Office \& Lab); Fax: +86-029-85324013

Email: zpsheng@mail.xjtu.edu.cn

\section{Abstract}

\section{Background}

Parental abnormal chromosomal karyotypes are considered as reasons for recurrent pregnancy loss.

\section{Objective}

This systematic meta-analysis evaluated the current evidence on pregnancy outcomes amongst couples with abnormal versus normal chromosomal karyotypes.

\section{Search strategy}

Two independent reviewers screened titles and abstracts identified in EMBASE and PubMed from inception to January 2021.

\section{Selection criteria}

Studies were included if they provided a description of pregnancy outcomes of parental chromosomal abnormality.

\section{Data collection and analysis}

Random effects meta-analysis was used to compare odds of pregnancy outcomes associated with noncarriers and carriers.

\section{Main results}

A significantly lower first pregnancy live birth rate (FPLBR) was found in carriers than in noncarriers with RPL (OR: 0.55; 95\% CI: 0.46-0.65; p<0.00001). Regarding FPLBR between translocation or inversion carriers and noncarriers, a markedly decreased FPLBR was found in translocation (OR: 0.44; 95\% CI: 0.31$0.61 ; \mathrm{p}<0.00001)$ but not inversion carriers. The accumulated live birth rate (ALBR) (OR: 0.96; 95\% CI: $0.90-1.03 ; \mathrm{p}=0.26)$ was similar, while the miscarriage rate $(\mathrm{MR})$ of accumulated pregnancies (OR: $2.21 ; 95 \%$ CI: $1.69-2.89 ; \mathrm{p}<0.00001)$ was significantly higher in the carriers than in noncarriers with RPL. The ALBR was not significant (OR: 1.82; 95\% CI: 0.38-8.71; $\mathrm{p}=0.45$ ) but the MR (OR: $5.75 ; 95 \%$ CI: 2.57-12.86; $\mathrm{p}<0.0001)$ was markedly lower for carriers who choose PGD than natural conception.

\section{Conclusions}

Carriers with RPL had higher risk of miscarriage but obtained a satisfying pregnancy outcome through multiple attempts. No sufficient evidence was found PGD could enhance the ALBR but it was an alternative to decrease the MR.

KEY WORDS: recurrent pregnancy loss / abnormal karyotype / pregnancy outcomes / PGD

\section{Introduction}

Recurrent pregnancy loss (RPL) is defined as two or more pregnancy losses before 20 weeks with a 1-3\% incidence rate from ESHRE in 2018 [1]. In addition to unexplained miscarriage accounting for 50\% of RPL [2], other factors, such as female age, uterine anomalies, endocrine disturbances, autoimmune antibodies, thrombophilia and chromosomal aberrations, are known risks of RPL[3]. Parental chromosomal aberrant couples, called carriers, account for $2-4 \%$ of the recurrent pregnancy loss population[4, 5]. Unbalanced gametes generated from chromosomal aberrant parents are associated with RPL, but no direct causal relationship has been proven between the parental chromosome and RPL, because foetal chromosomal abnormalities of 
abortion tissue are common not only in carriers but also in noncarriers[6, 7]. No consensus exists regarding the difference in pregnancy outcomes between carriers and noncarriers with RPL to date. Additionally preimplantation genetic diagnosis (PGD) has been performed increasingly at several centres worldwide, and has been applied for patients with chromosomal aberrations. Can PGD improve live birth rates compared with natural conception or expectant management (NC or EM) in RPL carriers? However, no evidence has substantiated the reproductive outcome benefit of these carriers after EM or after PGD. Recurrent pregnancy loss with abnormal chromosomes is a frustrating challenge for clinicians because no convincing evidence of effective therapy exists for these patients. The present study aimed to systematically analyse the best evidence from the efficacy literature to illustrate these associative questions.

\section{Methods}

\section{Search strategy}

EMBASE (1980 to January 2021) and PubMed (1950 to January 2021) were systematically searched as well as the reference lists of the selected articles. Initially, a search was conducted for comparative studies comparing the pregnancy outcomes in the carriers and noncarriers with RPL. Next, a search was conducted to compare the pregnancy outcomes after attempting EM with those after performing PGD in the RPL carriers. 'Recurrent pregnancy loss' , 'chromosome abnormalities', 'reproductive outcome','parental' and 'preimplantation genetic diagnosis' were used as keywords in the searches performed by a clinical librarian (JJ.H.). The appendix shows the search strategies in EMBASE, and adapted for PubMed, which were used to investigate the pregnancy outcomes between carriers and noncarriers and after EM or PGD in RPL carriers.

\section{Selection criteria and Study selection}

Studies were selected if the pregnancy outcomes of RPL couples with abnormal chromosomal were reported. RPL couples had two or three more miscarriages before 20 weeks. Carriers were defined as the chromosomal abnormalities in any one of the couples with RPL. Chromosomal abnormalities included structural and numerical abnormalities and excluded normal polymorphisms such as pstk, qh and pss. Karyotype analysis was the technological means used to distinguish carriers and noncarriers. The PGD was performed in a polar body biopsy or blastomere biopsy using either fluorescent in situ hybridization (FISH), comparative genomic hybridization (aCGH) or a single nucleotide polymorphism array (SNP) array. The primary outcome was the live birth rate (LBR) per couple, and secondary outcome was the miscarriage rate per couple. All the cohort studies described the pregnancy outcomes after natural conception or after PGD for carriers and noncarriers. Two independent investigators (S.L. and JJ.H.) extracted the data and compared the results, and all disagreements were resolved by discussion.

\section{Statistical analysis}

All the study results were presented as an odds ratio (OR) and $95 \%$ CI to reach a consistent data. $\mathrm{I}^{2}$ was used to access the heterogeneity in the meta-analysis. A random effect model was performed if the $\mathrm{I}^{2}$ measurement was greater than $50 \%$, and a fixed effect model was performed if $\mathrm{I}^{2}$ was less than $50 \%$. RevMan (Review Manager 5, version 5.3. Copenhagen: The Nordic Cochrane Centre, The Cochrane Collaboration, 2014) was used in the meta-analyses. The Newcastle-Ottawa scale was used to assess the quality of the studies.

\section{Results}

Results of the search

The flow chart of the study is presented in Figure S1. After rejecting articles not associated with the research question, 11 articles were included in the search for studies describing pregnancy outcomes after attempting natural conception in recurrent miscarriage couples from 96 publications, and 2 articles were included in the search for studies reporting on reproductive outcomes after EM or PGD in RPL carriers. Eleven comparative studies (including PS Zheng 2021, our pending published data) compared the first and/or accu- 
mulated pregnancy outcomes of carriers to the pregnancy outcomes of noncarriers after attempting natural conception[8-17]. Two comparative studies compared the pregnancy outcomes of carriers after attempting EM or PGD $[18,19]$. The population characteristics of the 13 included studies are shown in the Table 1.

Comparison of the pregnancy outcomes of carriers with noncarriers in couples with RPL

Eleven studies $(\mathrm{n}=6301)$ reported on the difference in the pregnancy outcomes of carriers versus noncarriers with a history of recurrent pregnancy loss. Eight studies and our own data (PS Zheng 2021) involved both structural and numerical chromosome abnormalities [9, 10, 12-16] and only 3 studies involved single structural chromosome abnormalities[8,11, 17]. All the studies comprised couples in which one partner had a chromosome abnormality. All the studies evaluated the pregnancy outcome of conceiving naturally in both carriers and noncarriers. Karyotype analysis was the main method used in all the included studies.

When pooling the studies, we found a significant difference in the first pregnancy live birth rate (FPLBR) for couples with RPL between carriers and noncarriers (nine studies; odds ratio (OR): 0.55; 95\% CI: 0.46-0.65; $\mathrm{I}^{2}=27 \% ; \mathrm{p}<0.00001$ ) (Figure 1a). When summing the individual proportions in the 9 studies, we found a mean prevalence of $55.6 \%$ (range: $25.0 \%-75.0 \%$; 95\% CI: $0.44-0.67$ ) in non-carriers and $67.8 \%$ (range: $35.7 \%-82.6 \%$; $95 \%$ CI $0.57-0.79$ ) in carriers of FPLBR. The detailed prevalence in each study is shown in the Table 2.

When summarizing chromosomally abnormal subtype proportions in the studies using meta-analysis, we found a significantly lower of the FPLBR in translocation carriers (Balanced and Robertsonian, Figure 1b) but no difference was observed in inversion carriers (Figure 1c) compared with noncarriers (six studies; translocation odds ratio (OR): $0.44 ; 95 \%$ CI: $0.31-0.61 ; \mathrm{I}^{2}=33 \% ; \mathrm{p}<0.00001$; inversion odds ratio (OR): 0.74; 95\% CI: $\left.0.52-1.06 ; \mathrm{I}^{2}=0 \% ; \mathrm{p}=0.1\right)$. These results indicate that the decline in the FPLBR of carriers was primarily associated with translocation but not inversion couples. No publication bias was found in the 9 studies as shown in the funnel plot (Figure S2).

Only 4 studies described the accumulated LBR (ALBR) and miscarriage rate (MR). The ALBR was similar between carriers and noncarriers (Figure 2a; four studies; odds ratio (OR): 0.96; 95\% CI: 0.90-1.03; $\mathrm{I}^{2}=0 \%$; $\mathrm{p}=0.26$ ), but the miscarriage rate was still significantly higher in the carriers (Figure $2 \mathrm{~b}$; four studies; odds ratio (OR): $2.21 ; 95 \%$ CI: $1.69-2.89 ; \mathrm{I}^{2}=0 \% ; \mathrm{p}<0.00001$ ) in the natural conception way. The detailed prevalence in each study is shown in Table 3 and 4 . No publication bias was found in the 4 studies, as shown in the funnel plot (Figure S2).

Comparison of the pregnancy outcomes of natural conception with PGD in RPL carriers

Few studies have comparied the pregnancy outcomes after choosing between NC/EM (natural conception/expectant management) and PGD for RPL carriers, because randomized control trials are unavailable. We systemically searched the literature as much as possible for the available evidence on pregnancy outcomes to describe this question and only 2 nonrandomized comparative studies comparing the pregnancy outcomes after PGD with those after NC in our study (Table 5). Savanie reported that $74 \%$ of RPL carriers with NC and $38.5 \%$ of those with PGD had a live birth, although the period of follow-up was significantly longer in the NC group ( $\mathrm{p}=0.016$ )[19]. Shinichiro reported $37-63 \%$ on the first trial, $65-83 \%$ cumulatively with NC and 27-54\% with PGD had at least a live birth[18]. The meta-analysis showed no significant difference in the accumulated LBR (Figure 3a; two studies; odds ratio (OR): 1.82; 95\% CI: 0.38-8.71; $\mathrm{I}^{2}=71 \%$; $\mathrm{p}=0.45$ ) but a higher miscarriage rate (Figure 3b; two studies; odds ratio (OR): $5.75 ; 95 \%$ CI: 2.57-12.86; I ${ }^{2}=45 \%$; $\mathrm{p}<0.0001)$ when comparing NC with PGD in RPL carriers.

\section{Discussion}

Chromosomal abnormalities in couples with recurrent pregnancy loss accounted for $3.75 \%$ in our unpublished data, consistent with 2\%-5\% found in other studies, representing a 5-10 fold increase compared with that in the general population $[4,20]$. Structural chromosomal abnormalities can lead to unbalanced gametes depending on specific recombination and segregation patterns during meiosis. The attendant reproductive risk depends on the type of rearrangement and its parental origin[21]. Some experts considered no significant 
impact of parental aberrant chromosomes on the pregnancy outcomes $[9,12,15]$ while others did not $[10,11$, 13, 14]. Additionally, Maureen Franssen analysed the live birth rate per couple of one time and more times pregnancies separately, and found a difference in the FPLBR and ALBR accordingly[17]. No coinciding views of the influence of parental chromosomal aberration on the pregnancy outcomes were found, such as live birth or miscarriage risk. The divergence in the above studies has prompted us to perform a meta-analysis to determine the real effect on the pregnancy outcomes of carriers and no similar earlier meta-analysis was found in the present literature. Generally, parental chromosomal aberration significantly increased the risk of miscarriage and decreased FPLBR in various populations of patients in this meta-analysis.

The innovation of the present meta-analysis compensates for the deficiency in the literature as follows: first, as the chance of finding an abnormality is very low, some previous studies did not obtain reliable results because of an insufficient sample size. More than 700 carriers and 5000 noncarriers were included from European, Asian, and North American countries. Second, chromosomal aberrant subtypes, such as translocation, inversion or numerical aberration were not calculated separately, while the different types had different degrees of influence on the pregnancy outcomes $[9,12,15]$. Subtype analysis could obtain a significant result more likely according to the different effects on the development of foetus. Finally, the live birth rate of one time or multiple pregnancies was not distinguished and unified in some studies. A higher live birth rate of one pregnancy indicates a lower miscarriage rate and vice versa, while this relationship does not exist between the live birth rate of multiple pregnancies and the miscarriage rate per couple. The data on the LBR and miscarriage rate per couple were processed uniformly in our study.

To determine real effect of parental chromosomal aberrations on the live birth and miscarriage rate, this systematic review investigated the available literature on pregnancy outcomes in recurrent miscarriage couples with parental chromosomal abnormalities and normalities. Different populations and areas were also included. Heterogeneity $\left(\mathrm{I}^{2}\right)$ was no more than $50 \%$ in all the results. Overall, we found that parental chromosomal abnormalities increased the risk of miscarriage in couples with RPL, the initial live birth rate was significantly lower, particularly for the translocation carriers but not inversion carriers. However, these RPL carriers eventually achieved a satisfactory accumulated live birth rate per couple, a finding that agreed with a early study[22]. Natural conception should be considered a good alternative because its accumulated live birth rate is satisfactory for carriers with RPL. Carriers have a risk of miscarriage because of abnormal gametes, but can obtain a live child through trials of multiple pregnancies. Because carriers with translocations are more likely to produce unbalanced gametes than inversions, translocations may have a larger effect on miscarriage.

ESHRE recommends against performing routine karyotyping of RPL couples, but rather after an individual risk assessment such as female age [1]. Once chromosome aberrations are detected in RPL couples who are confronted with chaenging decisions, such as trying to naturally conceive again, undergoing amniocentesis in future pregnancies and terminating a pregnancy once an unbalanced foetal karyotype is found or performing IVF/PGD and choosing normal karyotype embryos to transfer. The treatment of chromosome abnormalities in RPL couples comprises PGD in many reproductive centres worldwide. Although PGD seems an attractive and promising option for couples who are desperate for help, the benefits of PGD have been inconclusive thus far before introducing this technique into our routine medical work. Some studies seemed to illustrate strong evidence for a significant association between the pregnancy outcomes of PGD in carriers and showed that PGD improved the outcomes[23, 24]. These studies were doubtful and equivocal because they compared the results before and after the PGD for carriers and could not exclude clinical management in addition to the PGD. Non-genetic treatment is valuable and the main intervention in the natural conception for carriers with recurrent miscarriage, such as immunotherapy[25].

PGD has been proposed to improve and optimize live birth since 1988 and has been used to identify genetic abnormalities before embryo transfer[26]. Thousands of cycles have been performed worldwide and partial studies have indicated that PGD could increase successful pregnancy outcomes in translocation carriers by selecting balanced embryos[27, 28]. However, limited evidence in couples with RPL shows definite benefits of PGD or natural conception. Three earlier systematic reviews of PGD did not show an advantage with this 
strategy compared with expectant management and no sufficient evidence showed that PGD improves the pregnancy outcomes in RPL carriers, compared with NC[29-31]. The above three meta-reviews only list some studies about the pregnancy outcomes of using PGD alone or using NC alone, without comparative studies included. In our data we found no significant difference in the accumulated LBR but a higher miscarriage rate comparing $\mathrm{NC}$ with $\mathrm{PGD}$ in RPL carriers in two comparative outcomes. PGD could help decrease miscarriage risk, but failed or canceled cycles with no normal embryos to transfer, IVF related complications and high cost are main concerns for patients considering PGD[32].

The quantity and quality of the evidence on the comparing pregnancy outcomes between the PGD and natural conception were low. The alternatives for patients are affected by various subjective and objective factors; thus we could hardly compare the benefits of PGD and natural conception randomly. According to the above situation, we should be very cautious before making any conclusions or recommendations to clinical patients. Doctors must recongnize that the pregnancy outcome of recurrent abortion is caused by many factors, includingunknown factors, and cannot regard any an abnormal result as the factual relationship of abortion.

The origin of the heterogeneity in the present review was not found among the included studies because the female age and miscarriage times were similar before diagnosis. Other factors, such as diversity in ethnicity, the type of translocation, PGD methods and study design, might be sources of heterogeneity without adjusting the data for these factors, representing a potential limitation of this study. Another limitation of our meta-analysis was the insufficient number of studies, particularly for subgroup analysis and PGD analysis.

Conclusion

The results of this systematic review may support that RPL carriers have a higher risk of miscarriage than noncarriers. Thus the live birth rate of one pregnancy is lower,but carriers can eventually obtain a satisfactory cumulative live birth rate through multiple pregnancies. Furthermore, additional studies of the benefits of PGD in the RPL carriers are urgently required.

Study funding: This work was supported by the National Key Research and Development Program of China (No. 2018YFC1002804), the National Natural Science Foundation of China (No. 81901497).

Conflict: All authors declare that they have no conflict of interest in the article.

1. Bender Atik, R., et al., ESHRE guideline: recurrent pregnancy loss. Hum Reprod Open, 2018. 2018 (2): p. hoy004.

2. Alijotas-Reig, J. and C. Garrido-Gimenez, Current concepts and new trends in the diagnosis and management of recurrent miscarriage.Obstet Gynecol Surv, 2013. 68 (6): p. 445-66.

3. Jauniaux, E., et al., Evidence-based guidelines for the investigation and medical treatment of recurrent miscarriage. Hum Reprod, 2006. 21 (9): p. 2216-22.

4. Popescu, F., C.R. Jaslow, and W.H. Kutteh, Recurrent pregnancy loss evaluation combined with 24chromosome microarray of miscarriage tissue provides a probable or definite cause of pregnancy loss in over 90\% of patients. Hum Reprod, 2018. 33 (4): p. 579-587.

5. Fryns, J.P. and G. Van Buggenhout, Structural chromosome rearrangements in couples with recurrent fetal wastage. Eur J Obstet Gynecol Reprod Biol, 1998. 81 (2): p. 171-6.

6. Ogasawara, M., et al., Embryonic karyotype of abortuses in relation to the number of previous miscarriages. Fertil Steril, 2000.73 (2): p. 300-4.

7. Carp, H., et al., Karyotype of the abortus in recurrent miscarriage. Fertil Steril, 2001. 75 (4): p. 678-82.

8. FitzSimmons, J., et al., Subsequent reproductive outcome in couples with repeated pregnancy loss. Am J Med Genet, 1983.16 (4): p. 583-7. 
9. Carp, H., et al., Parental karyotype and subsequent live births in recurrent miscarriage. Fertil Steril, 2004. 81 (5): p. 1296-301.

10. Sugiura-Ogasawara, M., et al., Subsequent pregnancy outcomes in recurrent miscarriage patients with a paternal or maternal carrier of a structural chromosome rearrangement. J Hum Genet, 2008.53 (7): p. $622-628$.

11. Ozawa, N., et al., Pregnancy outcomes of reciprocal translocation carriers who have a history of repeated pregnancy loss.Fertil Steril, 2008. 90 (4): p. 1301-4.

12. Kabessa, M., et al., Pregnancy outcomes among patients with recurrent pregnancy loss and chromosomal aberration (CA) without PGD. J Perinat Med, 2018. 46 (7): p. 764-770.

13. Dong, Y., et al., Reproductive outcomes in recurrent pregnancy loss associated with a parental carrier of chromosome abnormalities or polymorphisms. Genet Mol Res, 2014. 13 (2): p. 2849-56.

14. Sugiura-Ogasawara, M., et al., Poor prognosis of recurrent aborters with either maternal or paternal reciprocal translocations.Fertil Steril, 2004. 81 (2): p. 367-73.

15. Pal, S., et al., Chromosomal abnormalities and reproductive outcome in Malaysian couples with miscarriages. Singapore Med J, 2009.50 (10): p. 1008-12.

16. Flynn, H., et al., Comparison of reproductive outcome, including the pattern of loss, between couples with chromosomal abnormalities and those with unexplained repeated miscarriages. J Obstet Gynaecol Res, 2014. 40 (1): p. 109-16.

17. Franssen, M.T., et al., Reproductive outcome after chromosome analysis in couples with two or more miscarriages: index [corrected]-control study. Bmj, 2006. 332 (7544): p. 759-63.

18. Ikuma, S., et al., Preimplantation Genetic Diagnosis and Natural Conception: A Comparison of Live Birth Rates in Patients with Recurrent Pregnancy Loss Associated with Translocation. PLoS One, 2015.10 (6): p. e0129958.

19. Maithripala, S., et al., Prevalence and Treatment Choices for Couples with Recurrent Pregnancy Loss Due to Structural Chromosomal Anomalies. J Obstet Gynaecol Can, 2018. 40 (6): p. 655-662.

20. Elkarhat, Z., et al., Chromosomal abnormalities in couples with recurrent spontaneous miscarriage: a 21-year retrospective study, a report of a novel insertion, and a literature review. J Assist Reprod Genet, 2019. 36 (3): p. 499-507.

21. Kaser, D., The Status of Genetic Screening in Recurrent Pregnancy Loss. Obstet Gynecol Clin North Am, 2018. 45 (1): p. 143-154.

22. Stephenson, M.D. and S. Sierra, Reproductive outcomes in recurrent pregnancy loss associated with a parental carrier of a structural chromosome rearrangement. Hum Reprod, 2006. 21 (4): p. 1076-82.

23. Fischer, J., et al., Preimplantation genetic diagnosis (PGD) improves pregnancy outcome for translocation carriers with a history of recurrent losses. Fertil Steril, 2010. 94 (1): p. 283-9.

24. Huang, C., et al., Pregnancy outcomes of reciprocal translocation carriers with two or more unfavorable pregnancy histories: before and after preimplantation genetic testing. J Assist Reprod Genet, 2019. 36 (11): p. $2325-2331$.

25. Carp, H., Immunotherapy for recurrent pregnancy loss. Best Pract Res Clin Obstet Gynaecol, 2019. 60 : p. $77-86$.

26. Munné, S., et al., First pregnancies after preconception diagnosis of translocations of maternal origin. Fertil Steril, 1998.69 (4): p. 675-81. 
27. Otani, T., et al., Preimplantation genetic diagnosis significantly improves the pregnancy outcome of translocation carriers with a history of recurrent miscarriage and unsuccessful pregnancies.Reprod Biomed Online, 2006. 13 (6): p. 869-74.

28. Lee, E., et al., The clinical effectiveness of preimplantation genetic diagnosis for aneuploidy in all 24 chromosomes (PGD-A): systematic review. Hum Reprod, 2015. 30 (2): p. 473-83.

29. Franssen, M.T., et al., Reproductive outcome after PGD in couples with recurrent miscarriage carrying a structural chromosome abnormality: a systematic review. Hum Reprod Update, 2011.17 (4): p. 467-75.

30. Musters, A.M., et al., Pregnancy outcome after preimplantation genetic screening or natural conception in couples with unexplained recurrent miscarriage: a systematic review of the best available evidence. Fertil Steril, 2011. 95 (6): p. 2153-7, 2157.e1-3.

31. Iews, M., et al., Does preimplantation genetic diagnosis improve reproductive outcome in couples with recurrent pregnancy loss owing to structural chromosomal rearrangement? A systematic review.Reprod Biomed Online, 2018. 36 (6): p. 677-685.

32. Schmutzler, A.G., Theory and practice of preimplantation genetic screening (PGS). Eur J Med Genet, 2019. 62 (8): p. 103670.

Figure 1 Comparison of the first pregnancy live birth rate (FPLBR) between carriers and noncarriers with recurrent pregnancy loss. a. FPLBR in all the carriers and non-carriers. $b$. FPLBR in translocations and non-carriers. c. FPLBR in inversion and non-carriers.

Figure 2 Comparison of the accumulated live birth rate (ALBR) and miscarriage rate (MR) between carriers and noncarriers with recurrent pregnancy loss. a. ALBR in carriers and non-carriers. b. MR in the carriers and non-carriers.

Figure 3 Comparison of the accumulated live birth rate (ALBR) and miscarriage rate (MR) between choosing expectant management (EM) and PGD in carriers with recurrent pregnancy loss. a. ALBR in the EM and the PGD. b. MR in the EM and the PGD.

Figure S1 Flowchart for the selection of studies

Figure S2 Funnel plot analysis (related to Fig 1).

Figure S3 Funnel plot analysis (related to Fig 2). 
a

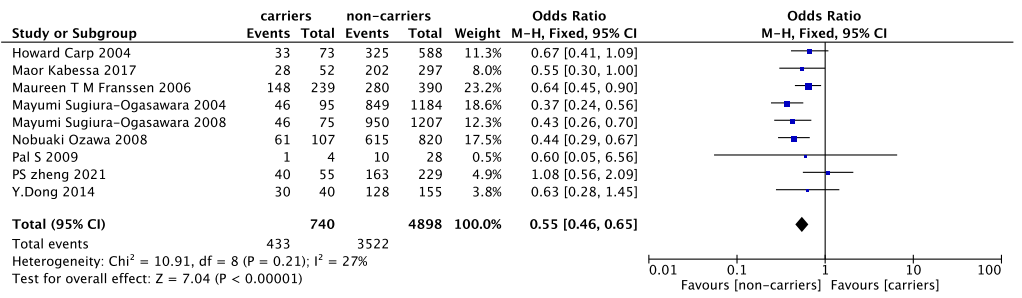

b

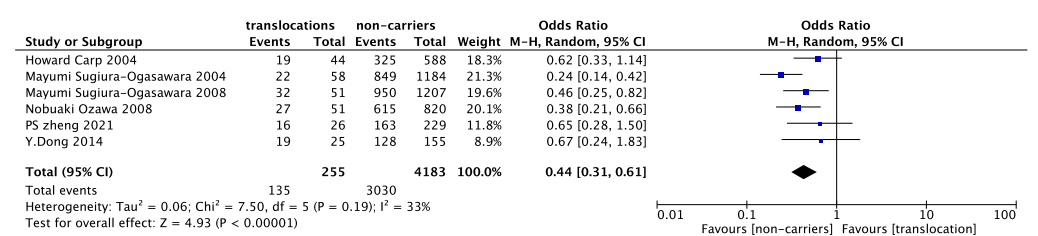

C

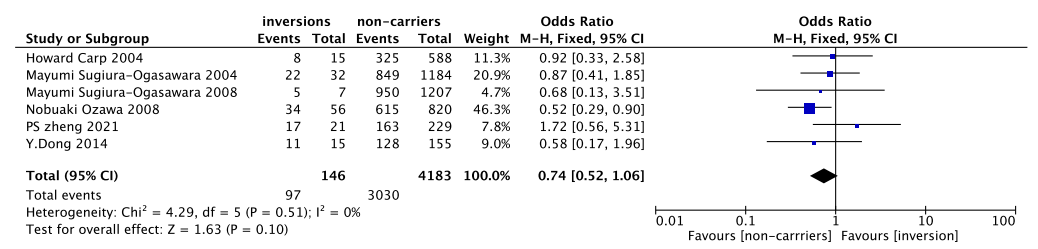

a

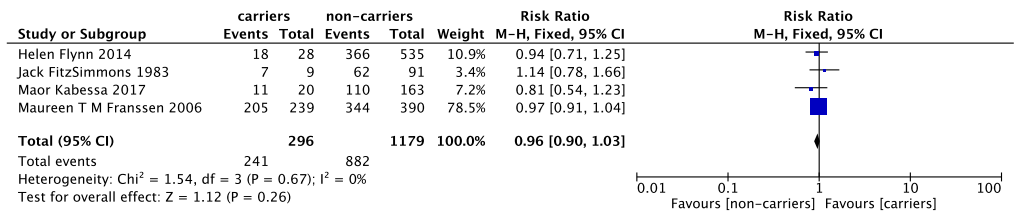

b

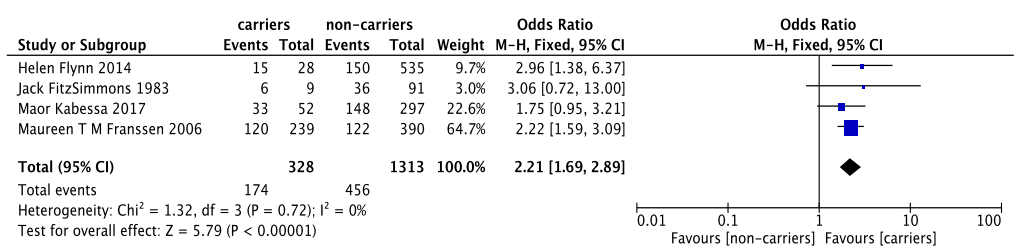


a

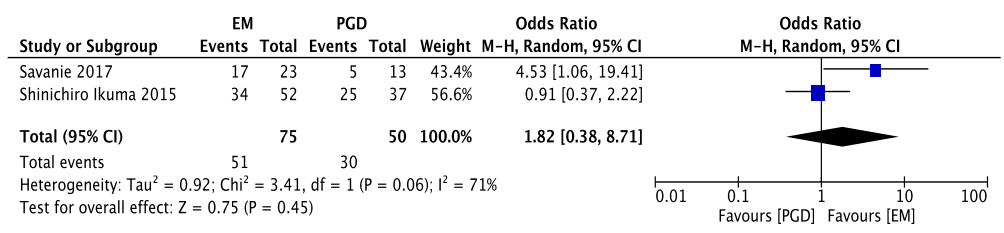

$\mathrm{b}$

\begin{tabular}{|c|c|c|c|c|c|c|c|c|c|}
\hline \multirow{2}{*}{$\begin{array}{l}\text { Study or Subgroup } \\
\text { Savanie } 2017\end{array}$} & \multirow{2}{*}{$\begin{array}{r}\text { EM } \\
\text { Events } \\
19\end{array}$} & Total & \multicolumn{2}{|c|}{$\begin{array}{c}\text { PGD } \\
\text { Events Total }\end{array}$} & \multirow{2}{*}{ Weight } & $\begin{array}{c}\text { Odds Ratio } \\
\text { M-H, Fixed, } 95 \% \mathrm{CI}\end{array}$ & \multicolumn{3}{|c|}{$\begin{array}{c}\text { Odds Ratio } \\
\text { M-H, Fixed, } 95 \% \mathrm{Cl}\end{array}$} \\
\hline & & 23 & 3 & 13 & & $15.83[2.95,85.08]$ & & & \\
\hline Shinichiro Ikuma 2015 & 30 & 52 & 9 & 37 & $87.0 \%$ & $4.24[1.67,10.76]$ & & & \\
\hline Total $(95 \% \mathrm{Cl})$ & & 75 & & 50 & $100.0 \%$ & $5.75[2.57,12.86]$ & & & \\
\hline Total events & 49 & & 12 & & & & & & \\
\hline \multicolumn{7}{|c|}{$\begin{array}{l}\text { Heterogeneity: } \mathrm{Chi}^{2}=1.80, \mathrm{df}=1(P=0.18) ; I^{2}=45 \% \\
\text { Test for overall effect: } Z=4.27(P<0.0001)\end{array}$} & 0.01 & $\begin{array}{c}1 \\
0.1 \\
\text { Favours [PCD] }{ }^{1} \mathrm{~F}\end{array}$ & Favours \\
\hline
\end{tabular}

\section{Hosted file}

Figure S1.pdf available at https://authorea.com/users/441170/articles/541733-systematicmeta-analysis-of-subsequent-pregnancy-outcomes-in-recurrent-pregnancy-loss-couples-withparental-abnormal-chromosomal-karyotype 


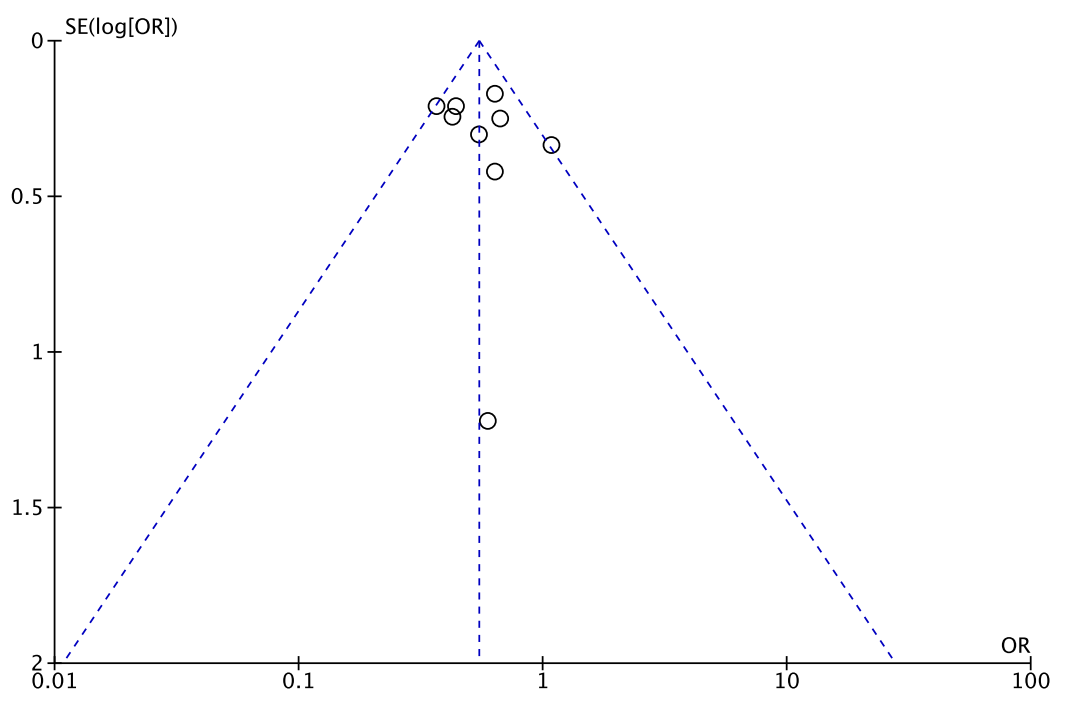




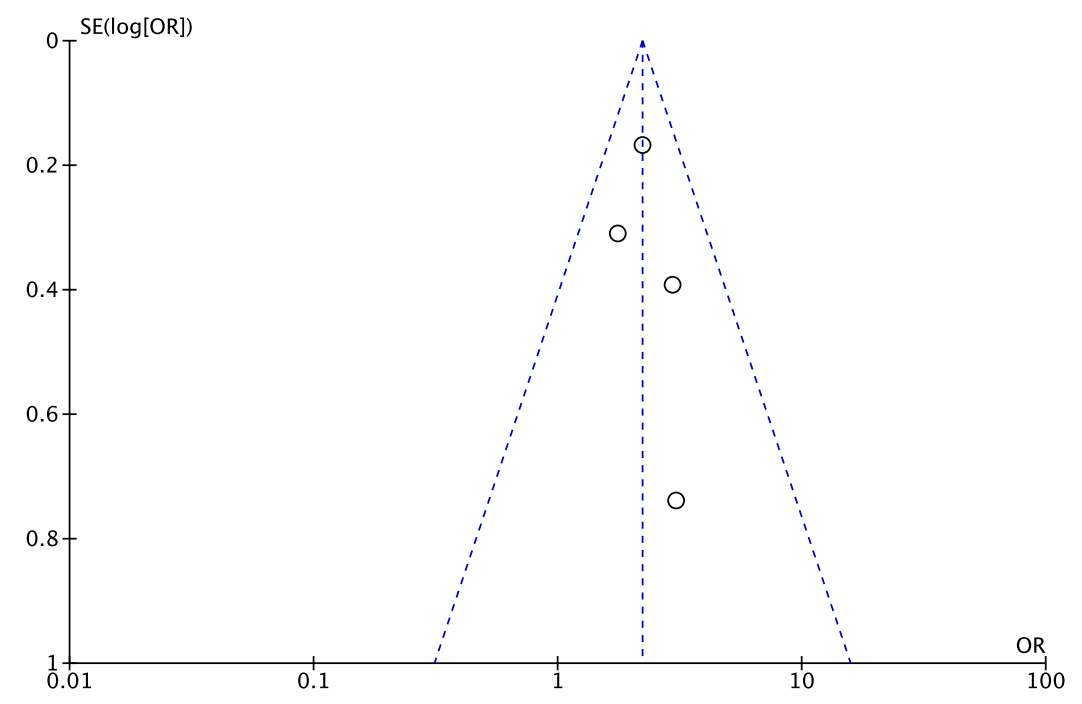

